\title{
ÉPOCA DE APLICAÇÃO DE NITROGÊNIO NO FEIJOEIRO IRRIGADO MONITORADA COMAUXÍLIO DE SENSOR PORTÁTIL
}

\author{
Timing of nitrogen application on irrigated common bean measured by portable sensor
}

\author{
Morel Pereira Barbosa Filho ${ }^{1}$, Tarcísio Cobucci ${ }^{1}$, Nand Kumar Fageria ${ }^{1}$, Patrícia Neves Mendes $^{2}$
}

\begin{abstract}
RESUMO
A definição da melhor época para aplicação de $\mathrm{N}$ nas culturas é fundamental para aumentar a eficiência de uso do $\mathrm{N}$ e a produtividade e reduzir custos com fertilizantes. Objetivou-se, neste trabalho avaliar formas e épocas de aplicação de $\mathrm{N}$ no feijoeiro. As técnicas de manejo consistiram em antecipar a aplicação do $\mathrm{N}$ em relação à semeadura, em aplicações baseadas na recomendação local e na suficiência de $\mathrm{N}$ avaliada por meio do teor de clorofila na folha, determinado com auxílio do clorofilômetro Minolta SPAD502. As produtividades de grãos alcançadas com a aplicação antecipada do $\mathrm{N}$ foram semelhantes àquelas obtidas em outras épocas. A aplicação de $\mathrm{N}$ na época indicada pelo ISN $<90 \%$ foi promissora em predizer a necessidade de aplicação de $\mathrm{N}$ em cobertura com gasto de $60 \mathrm{~kg} \mathrm{ha}^{-1} \mathrm{de} \mathrm{N}$ a menos e com maior eficiência agronômica do fertilizante. Os níveis críticos mínimos de leitura no clorofilômetro portátil para obtenção de $90 \%$ da produtividade máxima de grãos de feijão foram de 42 unidades-SPAD aos 30 dae e 46, no pleno florescimento do feijoeiro.
\end{abstract}

Termos para indexação: Phaseolus vulgaris L., manejo do nitrogênio, clorofilômetro, índice relativo de clorofila, índice de suficiência de nitrogênio.

\begin{abstract}
Defining the optimal timing of nitrogen application on a crop is fundamental to increase the efficiency of $\mathrm{N}$ use, increase the productivity and reduce the cost of fertilizers. The aim of this study was to evaluate methods and timing of $\mathrm{N}$ application for the common bean crop. The management technique consisted of $\mathrm{N}$ application previous to sowing, $\mathrm{N}$ application based on local recommendations, and based on the sufficiency of $\mathrm{N}$ evaluated through leaf chlorophyll content determined by the Minolta SPAD 502 Chlorophyll meter. Grain yield obtained with the application of $\mathrm{N}$ prior to sowing was similar to other periods of $\mathrm{N}$ applications. The nitrogen application indicated by ISN < 90\% was promising in predicting the necessity of $\mathrm{N}$ topdressing resulting in minus $60 \mathrm{~kg} \mathrm{~N} \mathrm{ha}^{-1}$ and higher agronomic efficiency of $\mathrm{N}$ use. Minimum critical levels of chlorophyll reading to obtain $90 \%$ maximum grain yield of dry bean were 42 unit-SPAD at 30 days after emergence and 46 days after emergence at full flowering of the bean plant.
\end{abstract}

Index terms: Phaseolus vulgaris L, Nitrogen management, chlorophyll meter.

(Recebido em 23 de março de 2007 e aprovado em 18 de outubro de 2007)

\section{INTRODUÇÃO}

O feijoeiro comum é a principal cultura em sistemas irrigados sob pivô central na região noroeste de Minas Gerais, com alto requerimento de nitrogênio $(\mathrm{N})$ e baixa eficiência de uso, sendo o sistema plantio direto o predominante na região. A semeadura geralmente é realizada sobre grande quantidade de resíduos vegetais deixados na superfície do solo pelas culturas antecessoras, e a adubação nitrogenada é muito influenciada por esses resíduos (BARBOSA FILHO et al., 2005a,b). Aproximadamente 52 mil hectares de feijão (LEVANTAMENTO..., 2006) são cultivados na região e uma das limitações referentes à prática de adubação é a falta de definição da melhor época para aplicação do $\mathrm{N}$ em cobertura, capaz de aumentar a eficiência de uso e reduzir o risco de contaminação ambiental e os custos com a aquisição de fertilizantes.

$\mathrm{Na}$ região noroeste de Minas Gerais, predominantemente sob o bioma Cerrado, as recomendações atuais para o uso de $\mathrm{N}$ em cobertura seguem as estratégias de manejo tradicionais, onde a dose geralmente ao redor de $100 \mathrm{~kg} \mathrm{ha}^{-1}$, é baseada em curvas de resposta da cultura ao $\mathrm{N}$ e a época de aplicação em cobertura é pré-determinada, não levando em consideração, a fase de maior demanda do feijoeiro (BARBOSA FILHO \& SILVA, 1994, 2000; BARBOSA FILHO et al., 2004, 2005b).

Como alternativa aos métodos convencionais de adubação de cobertura, estudos de teste rápido com auxílio

\footnotetext{
${ }^{1}$ Engenheiros Agrônomos, Doutores, Pesquisadores - Empresa Brasileira de Pesquisa Agropecuária/EMBRAPA - Rodovia Goiânia a Nova Veneza, Km 12 - Zona Rural - Cx. P. 179 - 75375-000 - Santo Antônio de Goiás, GO - morel@cnpaf.embrapa.br; fageria@cnpaf.embrapa.br; cobucci@cnpaf.embrapa.br ${ }^{2}$ Estatística, Mestre - Departamento de Ciências Exatas/DEX - Universidade Federal de Lavras/UFLA - Cx. P. 3037 - $37200-000$ - Lavras, MG patyestatistica@yahoo.com.br - Bolsista CNPq
} 
de sensor portátil têm sido realizados com muito sucesso em vários países para monitorar o "status" de N em plantas e determinar a época mais adequada para a sua aplicação em diversas culturas (FURLANI JÚNIOR et al., 1996; HUSSAIN et al., 2000; PENG et al., 1993; PIEKIELEK \& FOX, 1992; STONE et al., 2002). Os resultados têm sido positivos apresentando, em geral, maior eficiência agronômica do $\mathrm{N}$ aplicado do que com as práticas convencionais, baseadas em épocas pré-fixadas para aplicação do $\mathrm{N}$ em cobertura.

O clorofilômetro portátil tem sido muito usado como instrumento indicador da necessidade de aplicação de N, por meio de relações de leituras do clorofilômetro e o conteúdo de $\mathrm{N}$ na planta e no solo (SCHARF et al., 2006). Porém, em sistemas de produção de sequeiro uma das limitações do clorofilômetro é não permitir quantificar a dose de $\mathrm{N}$ a ser aplicada no momento da tomada de decisão. Já em sistemas irrigados, há possibilidade de aplicação de pequenas doses na água de irrigação, toda vez que as leituras obtidas no aparelho caírem abaixo de um valor crítico pré-determinado (VARVEL et al., 1997). Por outro lado, nos sistemas de sequeiro, as aplicações de pequenas doses e mais freqüentes, são mais difíceis e podem não ser viáveis economicamente.

Objetivou-se, neste trabalho, comparar diferentes estratégias de manejo do $\mathrm{N}$ no que se refere às épocas para a aplicação de $\mathrm{N}$ em cobertura na cultura do feijoeiro irrigado, com auxílio do clorofilômetro portátil.

\section{MATERIALE MÉTODOS}

O experimento foi realizado de julho a setembro de 2004, na Fazenda Guaíba localizada no município de Unaí, MG em solo latossolo vermelho distroférrico. Utilizou-se o sistema plantio direto (SPD) em área coberta com palhada de milho e a cultivar de feijão usada foi a Pérola, semeada no espaçamento entrelinhas de $0,45 \mathrm{~m}$, e aproximadamente 12 sementes viáveis por metro linear de sulco. As parcelas mediam $10 \mathrm{~m}$ x $8 \mathrm{~m}$, sendo $9 \mathrm{~m}^{2}$ considerada como área útil. Antes da semeadura foi tomada uma amostra composta de solo que foi analisada, e os resultados revelaram: $\mathrm{pH}\left(\mathrm{H}_{2} \mathrm{O}\right)$, 5,6; P (Mehlich 1), 6,2 $\mathrm{mg} \mathrm{kg}^{-1}$; K trocável (Mehlich 1), 75 $\mathrm{mg} \mathrm{kg}^{-1} ; \mathrm{Ca}+\mathrm{Mg}$ trocáveis $(\mathrm{em} \mathrm{KCl} \mathrm{0,1} \mathrm{N}), 27,8 \mathrm{mmol}$ $\mathrm{cm}^{-3} ; \mathrm{Al}^{+3}, 1,0 \mathrm{mmol} \mathrm{cm}^{-3} ; \mathrm{CTC}_{\mathrm{pH}}$, calculada pela soma de $\mathrm{Ca}+\mathrm{Mg}+\mathrm{K}+\mathrm{H}+\mathrm{Al}, 78,2 \mathrm{mmol} \mathrm{cm}{ }^{-3}$; sendo o $\mathrm{H}+\mathrm{Al}$ extraídos em acetato de cácio (CaAc), V\%,36,1 e matéria orgânica, $22 \mathrm{~g} \mathrm{~kg}^{-1}$.

A adubação básica consistiu de $100 \mathrm{~kg} \mathrm{ha}^{-1}$ de $\mathrm{P}_{2} \mathrm{O}_{5}$ e $70 \mathrm{~kg} \mathrm{ha}^{-1}$ de $\mathrm{K}_{2} \mathrm{O}$ nas formas de superfosfato triplo e cloreto de potássio, respectivamente. $\mathrm{O}$ delineamento experimental foi o inteiramente casualizado com seis tratamentos, constituídos por técnicas de manejo do N, e cinco repetições. Os tratamentos com $\mathrm{N}$ avaliados foram as seguintes: M1 (tratamento de referência): aplicação de $240 \mathrm{~kg} \mathrm{ha}^{-1}$ de $\mathrm{N}$, sendo $90 \mathrm{~kg}$ aos 15 dias antes da semeadura juntamente com o dessecante glifosate $+30 \mathrm{~kg}$ no plantio + $60 \mathrm{~kg}$ aos 15 dias após emergência (dae) + 60 $\mathrm{kg}$ aos 30 dae, M2: $120 \mathrm{~kg} \mathrm{ha}^{-1}$, sendo $90 \mathrm{~kg}$ aos 15 dias antes do plantio + $30 \mathrm{~kg}$ no plantio; M3: $120 \mathrm{~kg} \mathrm{ha}^{-1}$, sendo $60 \mathrm{~kg}$ aos 15 dias antes do plantio $+30 \mathrm{~kg}$ no plantio +30 $\mathrm{kg}$ aos 15 dae; M4: aplicação de $30 \mathrm{~kg} \mathrm{ha}^{-1}$ de $\mathrm{N}$ no plantio e aplicação em cobertura baseada no uso do clorofilômetro Minolta SPAD-502, usando o índice de suficiência de $\mathrm{N}<90 \%$. M5: aplicação de $120 \mathrm{~kg} \mathrm{ha}^{-1}$ de $\mathrm{N}$ baseada na recomendação local, ou seja, $30 \mathrm{~kg} \mathrm{ha}^{-1} \mathrm{de}$ $\mathrm{N}$ no plantio + $45 \mathrm{~kg}$ aos 15 dae $+45 \mathrm{~kg}$ aos 30 dae; e M6: testemunha sem $\mathrm{N}$.

As leituras no clorofilômetro (unidades-SPAD-Soil

Plant Analysis Developement) foram tomadas semanalmente de três pontos da parcela. Em cada ponto, as leituras foram realizadas em seis plantas, sendo feitas cinco leituras por folha (segunda folha trifoliada completamente desenvolvida) somando 30 leituras, com um total de 90 por parcela.

Os valores de densidade ótica (unidades SPAD) foram usados para determinar o teor total de clorofila pela equação linear $\hat{y}=-0,152+0,0996 x$ determinada por Barnes et al. (1992). As concentrações de clorofila foram plotadas contra os respectivos valores de unidades-SPAD, determinadas nas mesmas folhas. $\mathrm{Na}$ análise de $\mathrm{N}$ foram utilizadas as mesmas folhas avaliadas para determinação do teor relativo de clorofila.

O critério de Cate \& Nelson (1971) de determinação de níveis críticos, foi adotado plotando-se no eixo da abscissa ( $x$ ) os valores de unidades-SPAD e na coordenada (y) as produtividades relativas (\%), calculadas pela divisão das médias dos rendimentos de grãos pela média do tratamento com a maior produtividade de grãos, em duas épocas de crescimento do feijoeiro onde, normalmente, ocorre a deficiência de $\mathrm{N}$, uma aos 30 dias após emergência e outra em pleno florescimento das plantas. Os valores críticos de unidades-SPAD foram determinados nos níveis de $90 \%$ de produtividade máxima, abaixo dos quais há indicação de que possa haver resposta à aplicação de N suplementar (CATE \& NELSON, 1971). 
que se apresentam, baseadas nos trabalhos de Cabezas et al. (2004), Ceretta et al. (2002), Pauletti \& Costa (2000) e Pöttker \& Wiethölter (2004), possivelmente estão relacionadas à menor demanda de $\mathrm{N}$ na fase inicial de crescimento e aos baixos teores de matéria orgânica nos solos tropicais, além do que o $\mathrm{N}$ pode ser influenciado por algum processo de perda, devido ao seu acúmulo no solo.

A estratégia de manejo de $\mathrm{N}$ monitorado com o auxílio do clorofilômetro portátil (M4), utilizada na expectativa de que pudesse melhorar o sincronismo entre a época de fornecimento do $\mathrm{N}$ e demanda da planta, foi promissora, uma vez que não houve no referido tratamento diminuição na produtividade de grãos e a eficiência agronômica da adubação nitrogenada foi maior, demonstrando que, com a aplicação de $60 \mathrm{~kg} \mathrm{ha}^{-1} \mathrm{de} \mathrm{N}$ a menos (Tabela 1), foi possível alcançar níveis de produtividade de grãos tão elevados quanto àqueles obtidos, seguindo a técnica de manejo convencional em que a época para aplicação do $\mathrm{N}$ em cobertura foi prédefinida (M5). Resultados similares foram relatados por Hussain et al. (2000) e Peng et al. (1993) para as culturas de milho e arroz irrigado.

Na Figura 1, pode-se comparar as duas estratégias de aplicação do N, uma baseada nas leituras diretas (IRC) e outra na suficiência de N (ISN\%) determinadas a partir de um valor de referência, em relação a uma área adubada com alta dose de $\mathrm{N}$ de forma a assegurar a não ocorrência de deficiência de N. Observa-se que, entre 30 e 37 dae das plântulas, os valores de IRC diminuíram, ao contrário do esperado, já que receberam uma suplementação de $30 \mathrm{~kg}$ $\mathrm{ha}^{-1}$ de $\mathrm{N}$ aos 30 dae. Não foi possível identificar uma causa para essa redução do teor de clorofila aos 37 dae, mas o fato das leituras terem sido tomadas após uma irrigação, ainda com as folhas umedecidas, pode ter influenciado as leituras no aparelho. Segundo Piekielek \& Fox (1992) e Turner \& Jund (1991), além da disponibilidade de $\mathrm{N}$ no instante da tomada da leitura, a uniformidade dos valores de leituras-SPAD podem, também, sofrer influências de outros fatores, tais como a espessura da folha, a posição da folha em que a leitura é tomada, deficiências de outros nutrientes, estresse causado por doenças, pragas e altas temperaturas, fatores esses que, em geral, reduzem os teores de clorofila nas folhas.

As unidades-SPAD ou índice relativo de clorofila (IRC) foram crescentes com o tempo, estabilizando-se a partir dos 51 dias após emergência (dae), época em que as plantas encontravam-se na fase de florescimento (Figura 1). Isso indica que, após essa fase de crescimento do feijoeiro, o teor de clorofila nas folhas se estabiliza, tornando-se ineficaz o uso do aparelho a partir dessa fase, como indicador da necessidade da aplicação de $\mathrm{N}$ em cobertura. Aos 30 dae das plântulas foi observado com auxílio do clorofilômetro portátil, ISN abaixo de $90 \%$ no tratamento M4, indicando, portanto, o momento e a necessidade de aplicação de $\mathrm{N}$ suplementar, razão pela qual foram aplicados mais $30 \mathrm{~kg} \mathrm{ha}^{-1}$ de $\mathrm{N}$ em cobertura ao lado das fileiras de feijão, totalizando $60 \mathrm{~kg} \mathrm{ha}^{-1}$ de $\mathrm{N}$, suficientes para atingir uma produtividade razoável de 2.429 $\mathrm{kg} \mathrm{ha}^{-1}$ de feijão, comparável com as produtividades obtidas ao nível de $120 \mathrm{~kg} \mathrm{ha}^{-1}$ de $\mathrm{N}$ aplicadas em outras épocas (Tabela 1).

Uma limitação do uso do clorofilômetro para efeito de aplicação suplementar de $\mathrm{N}$ é não permitir quantificar a dose de $\mathrm{N}$ a ser aplicada no momento da tomada de decisão. Porém, segundo Vervel et al. (1997), em sistemas irrigados
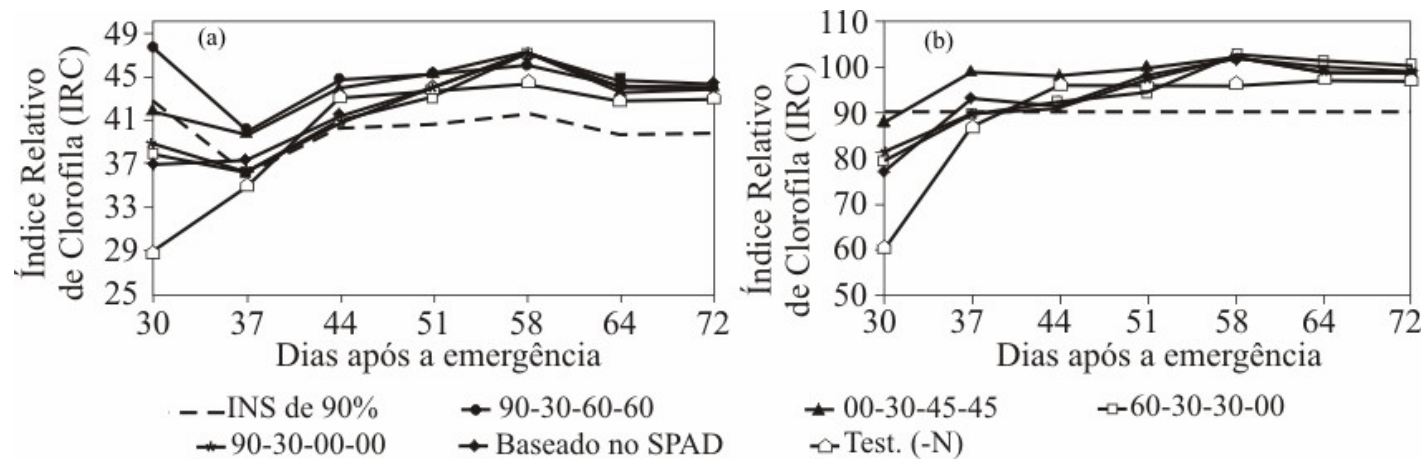

Figura 1 - Influência do manejo da adubação nitrogenada de cobertura sobre as leituras do clorofilômetro (unidadesSPAD) (a) e sobre o índice de suficiência de N (b) nas folhas do feijoeiro irrigado. O índice (ISN) de $90 \%$ representa $90 \%$ das leituras do tratamento de referência. 
existe a possibilidade de se aplicar o $\mathrm{N}$ em pequenas doses, e mais freqüentes via água de irrigação, toda vez que as leituras obtidas no aparelho caírem abaixo de um valor crítico pré-determinado, sem onerar significativamente o custo de produção.

$\mathrm{Na}$ fase inicial de crescimento do feijoeiro, observaram-se sintomas visuais de deficiência de $\mathrm{N}$ no tratamento sem aplicação de N (M4); porém a partir dos 42 dae, as plantas adquiriram a tonalidade verde, resultando em valores das unidades-SPAD muito próximas das leituras observadas nas parcelas adubadas com N (Figura 1). A explicação deste fato é devida ao processo de mineralização do $\mathrm{N}$ dos resíduos vegetais deixados na superfície do solo pelo cultivo anterior de milho, porém o $\mathrm{N}$ liberado durante o processo de mineralização não foi suficiente para suprir a necessidade do feijoeiro deste nutriente.

$\mathrm{O}$ fato das plantas tornarem-se verdes com o tempo de mineralização do $\mathrm{N}$ contido nos resíduos vegetais é indicação de que as unidades de leitura-SPAD podem ser afetadas, em função da quantidade e da qualidade dos resíduos vegetais antecedentes. Portanto, dependendo da época em que as unidades de leitura-SPAD são tomadas, podem ocorrer erros de interpretação, uma vez que podem não indicar deficiência do nutriente ocorrida na fase inicial de crescimento da planta, como foi constatada e comprovada a partir dos dados de produtividade de grãos apresentados na Tabela 1. Tomada essa precaução, podese considerar que o clorofilômetro Minolta SPAD-502 é promissor como instrumento indicativo da época quando se deve iniciar a aplicação de $\mathrm{N}$ em cobertura no feijoeiro irrigado. A adubação de cobertura baseada nesse critério resulta em maior EA do N, do que a aplicação de $\mathrm{N}$ baseada na recomendação local.

Segundo Piekielek \& Fox (1992), o fornecimento de $\mathrm{N}$ às plantas, seja por meio de aplicações de fertilizantes ou pela mineralização da matéria orgânica pode, temporariamente, compensar eventual deficiência de $\mathrm{N}$ e aumentar o teor de clorofila nas folhas no momento da tomada de leituras no aparelho. Isso é particularmente importante em áreas de predominância do SPD, em que os resíduos vegetais de cultivos anteriores são mantidos na superfície do solo. Nessas condições, em algum momento durante o crescimento da cultura sucessora, poderá ocorrer a liberação temporária de $\mathrm{N}$ para essa cultura da decomposição e mineralização dos resíduos vegetais e influenciar nas leituras do clorofilômetro.
Os teores de clorofila e a produtividade de grãos e $\mathrm{o}$ acúmulo de $\mathrm{N}$ nas folhas foram altamente relacionados (Figura 2a, b), possibilitando assim avaliar a necessidade de aplicação de $\mathrm{N}$ em cobertura no feijoeiro irrigado, o que confirma resultados de outros autores (BARNES et al., 1992; CARVALHO et al., 2003; FURLANI JÚNIOR et al., 1996; PENG et al., 1993). Os teores de clorofila e a produtividade de grãos aumentaram à medida que aumentou a absorção de N. Porém, ressalta-se que o teor de clorofila, pode variar com a espécie, cultivar e condições climáticas, especialmente radiação solar, deficiências de nutrientes, fase de crescimento da planta, posição da folha na planta, danos causados por doenças e pragas e práticas de manejo (PIEKIELEK \& FOX, 1992; TURNER \& JUND, 1991). Já a utilização do ISN\% em substituição às unidades de leitura-SPAD como indicador da necessidade de suplementação de $\mathrm{N}$ parece mais adequado, em função do melhor comportamento das curvas que expressam a relação entre as unidades de leitura-SPAD e o ISN\%, conforme se observa na Figura 1.

$\mathrm{O}$ fato de ter havido estreita relação entre as unidades de leitura-SPAD, teor de clorofila e produtividade relativa de grãos com $\mathrm{N}$ acumulado nas folhas (Figura 2a, b), requer a determinação de um nível crítico de unidades de leitura-SPAD que otimize o rendimento de grãos e a eficiência de uso do N. Aplicandose o método de Cate \& Nelson (1971) para determinação do nível crítico aos dados de produtividade relativa de grãos, estabeleceu-se o nível crítico de 42 unidades de leitura-SPAD aos 30 dae e de 46 aos 51 dae, em pleno florescimento das plantas (Figura 3a, b). Isso indica que, para valores acima desses referenciais, não há necessidade de suplementação de $\mathrm{N}$ e que a relação entre unidades de leitura-SPAD e teor de $\mathrm{N}$ nas folhas depende do estádio de crescimento da planta (PENG et al., 1993). Segundo Cate \& Nelson (1971), para uma técnica em estudo ser considerada adequada, todos os pontos deveriam situar-se dentro dos quadrantes inferior esquerdo e superior direito e aqueles que se encontram nos quadrantes inferior direito e superior esquerdo indicam quando o $\mathrm{N}$ suplementar de cobertura não deve ser recomendado (Figura 3a, b). Portanto, as unidades de leitura-SPAD abaixo do nível crítico de 42 aos 30 dae e 46 no pleno florescimento, resultantes em valores de produtividades situados no quadrante superior esquerdo indicam alta probabilidade de resposta à aplicação de $\mathrm{N}$ em cobertura pelo fato de as unidades de leitura-SPAD estarem abaixo do nível crítico, mas não se espera aumento da produtividade de grãos. 

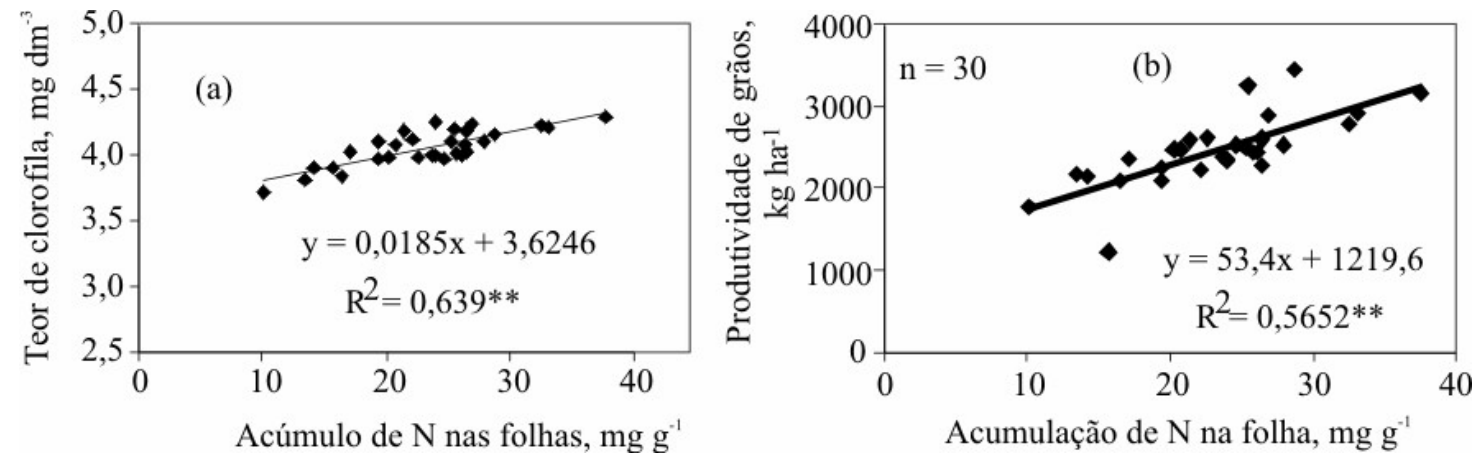

Figura 2 - Relação entre o teor de clorofila e acúmulo de $\mathrm{N}$ nas folhas (a) e entre a produtividade de grãos e a acumulação de $\mathrm{N}$ nas folhas na fase de florescimento pleno (51 dae) (b) do feijoeiro irrigado cultivado no sistema plantio direto, sob diferentes manejos de adubação nitrogenada de cobertura. **significativo a $1 \%$.
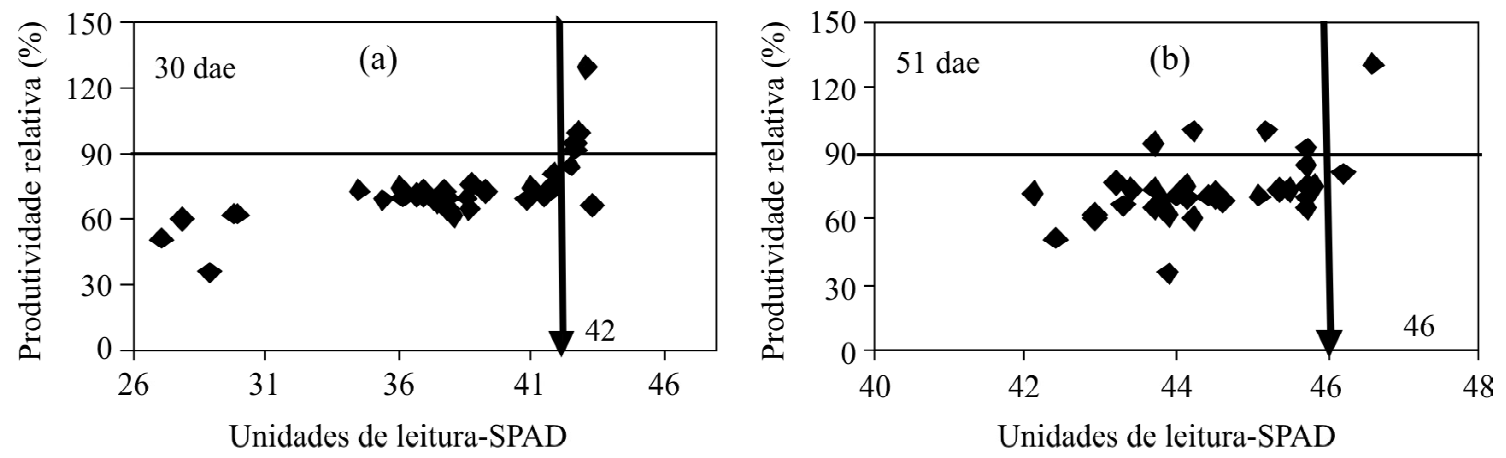

Figura 3 - Relação entre produtividade relativa de grãos e o índice relativo de clorofila (unidades de leitura-SPAD) e nível crítico de unidade de leitura-SPAD aos 30 dae (a) e na fase de florescimento pleno do feijoeiro irrigado (51dae) (b), segundo Cate \& Nelson (1971).

\section{CONCLUSÕES}

A estratégia de antecipar a aplicação de $\mathrm{N}$ em relação à semeadura, na adubação de cobertura do feijoeiro irrigado não foi superior à técnica de manejo de aplicação em outras épocas.

A aplicação de $\mathrm{N}$ na época indicada pelo ISN $<90 \%$ foi promissora em predizer a necessidade de aplicação de $\mathrm{N}$ em cobertura com gasto de $60 \mathrm{~kg} \mathrm{ha}^{-1} \mathrm{de} \mathrm{N}$ a menos e com eficiência agronômica do fertilizante maior.

Os níveis críticos mínimos de leitura no clorofilômetro portátil, para obtenção de produtividade de $90 \%$ da produtividade máxima de grãos de feijão foram de 42 unidades-SPAD aos 30 dae, e de 46 unidades-SPAD no pleno florescimento do feijoeiro.

\section{AGRADECIMENTOS}

Ao engenheiro agrônomo Luís Joaquim Missio, administrador da Fazenda Guaíba, pela concessão da área experimental e apoio na condução do experimento.

\section{REFERÊNCIAS BIBLIOGRÁFICAS}

BARBOSA FILHO, M. P.; COBUCCI, T.; MENDES, P. N. Adubação. In: Cultivo do feijão irrigado na

região noroeste de Minas Gerais. Disponível em: <http:/ i/sistemasdeprodução.cnptia.embrapa.br/FontesHTML/ Feijão/FeijaoIrrigadoNoroestêMG/, adubação.htmDez.2005i>. Acesso em: 25 out. $2005 \mathrm{a}$.

BARBOSA FILHO, M. P.; FAGERIA, N. K.; SILVA, O. F. da. Fontes, doses e parcelamento da adubação nitrogenada em cobertura para feijoeiro comum irrigado. Ciência e Agrotecnologia, Lavras, v. 29, n. 1, p. 69-76, $2005 b$.

BARBOSA FILHO, M. P.; FAGERIA, N. K.; SILVA, O. F. da. Fontes e métodos de aplicação de nitrogênio em feijoeiro irrigado submetido a três níveis de acidez do solo. Ciência e Agrotecnologia, Lavras, v. 28, n. 4, p. 785-792, 2004. 
BARBOSA FILHO, M. P.; SILVA, O. F. da. Adubação e calagem para o feijoeiro irrigado em solo de cerrado. Pesquisa Agropecuária Brasileira, Brasília, v. 35, n. 7, p. 1317-1324, 2000.

BARBOSA FILHO, M. P.; SILVA, O. F. da. Aspectos agroeconômicos da calagem e da adubação nas culturas de arroz e feijão irrigados por aspersão. Pesquisa Agropecuária Brasileira, Brasília, v. 29, n. 11, p. 16571667, 1994.

BARNES, J. D.; BALGUER, L.; MANRIQUE, E.; ELVIRA, S.; DAVISON, A. W. A reappraisal of the use of DMSO for the extraction and determination of chlorophylls a and $b$ in lichens and higher plants. Environmental and Experimental Botany, Oxford, v. 32, p. 85-100, 1992.

CABEZAS, L. W. A. R.; ALVES, B. J. R.; CABALLERO, S. S. U.; SANTANA, D. G. de. Influência da cultura antecessora e da adubação nitrogenada na produtividade de milho em sistema plantio direto e solo preparado. Ciência Rural, Santa Maria, v. 34, n. 4, p. 1005-1013, 2004.

CARVALHO, M. A. C.; FURLANI, E.; ARF, O.; SÁ, M. E.; PAULINO, H. B.; BUZETTTI, S. Doses e épocas de aplicação de nitrogênio e teores foliares deste nutriente e de clorofila em feijoeiro. Revista Brasileira de Ciência do Solo, Viçosa, v. 27, n. 3, p. 445-450, 2003.

CATE, R. B. J. R.; NELSON, L. A. A simple statistical procedure for partitioning soil correlation data into classes. Soil Science Society of America Proceedings, Madison, v. 35, p. 658-660, 1971.

CERETTA, C. A.; BASSO, C. J.; FLECHA, A. M. T.; PAVINATO, P. S.; VIEIRA, F. C. B.; MAI, M. E. M. Manejo da adubação nitrogenada na sucessão aveia preta/milho, no sistema plantio direto. Revista

Brasileira de Ciência do Solo, Viçosa, v. 26, n. 1, p. 163171, 2002.

FURLANI JÚNIOR, E.; NAKAGAWA, J.; BULHÕES, L. J.; MOREIRA, J. A. A.; GRASSI FILHO, H. Correlação entre leituras de clorofila e níveis de nitrogênio aplicados em feijoeiro. Bragantia, Campinas, v. 55, n. 1, p. 171-175, 1996.

HUSSAIN, F.; BRONSON, K. F.; SINGH, Y.; SINGH, B.; PENG, S. Use of chlorophyll meter sufficiency indices for nitrogen management of irrigated rice in Asia. Agronomy Journal, Madison, v. 92, n. 5, p. 875-879, 2000.

LEVANTAMENTO sistemático da produção agrícola. Rio de Janeiro, RJ: IBGE, 2006.

\section{MORAIS, J. F. V.; RABELO, N. A. Um método simples para a digestão de amostras de plantas. Goiânia:} Embrapa-CNPAF, 1986. 12 p. (Documentos, 12).

PAULETTI, V.; COSTA, L. C. Época de aplicação de nitrogênio no milho cultivado em sucessão à aveia preta no sistema plantio direto. Ciência Rural, Santa Maria, v. 30, n. 4, p. 599-603, 2000.

PENG, S.; GARCIA, F. V.; LAZA, R. C.; CASSMAN, K. G. Adjustment for specific leaf weight improves chlorophyll meter estimate of rice leaf nitrogen concentration.

Agronomy Journal, Madison, v. 85, n. 5, p. 987-990, 1993.

PIEKIELEK, W. P.; FOX, R. H. Use of a chlorophyll meter to predict sidedress nitrogen requirements for maize.

Agronomy Journal, Madison, v. 84, p. 59-65, 1992.

PÖTTKER, D.; WIETHÖLTER, S. Épocas e métodos de aplicação de nitrogênio em milho cultivado no plantio direto. Ciência Rural, Santa Maria, v. 34, n. 4, p. 10151020, 2004.

SCHARF, P. C.; BROUDER, M.; HOEFT, R. G.

Chlorophyll meter readings can predict nitrogen need and yield response of corn in the North-Central USA. Agronomy Journal, Madison, v. 98, p. 655-665, May/ June 2006.

STONE, L. F.; SILVA, G. de M.; MOREIRA, J. A. A. Uso do clorofilômetro SPAD-502 na estimativa do nitrogênio foliar específico e da produtividade do feijoeiro. In: CONGRESSO NACIONAL DE PESQUISA DE FEIJÃO, 7., 2002, Viçosa, MG. Resumos expandidos... Viçosa: UFV, 2002. p. 743-746.

TURNER, F. T.; JUND, M. F. Chlorophyll meter to predict nitrogen topdress requirement for semidwarf rice.

Agronomy Journal, Madison, v. 83, p. 926-928, 1991.

VERVEL, G. E.; SCHEPERS, J. S.; FRANCIS, D. D. Ability for in season correction of nitrogen deficiency in corn using chlorophyll meters. Soil Science Society of America Journal, Madison, v. 61, p. 1233-1239, 1997. 